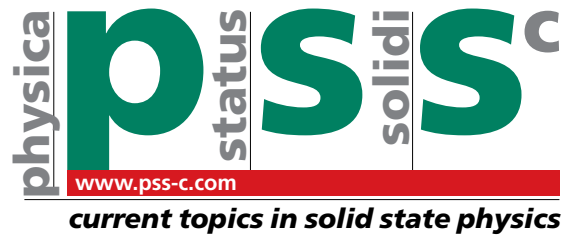

\title{
The water-like film on water
}

\section{R. Greef* and J. G. Frey}

School of Chemistry, Southampton University, Highfield, Southampton SO17 1BJ, UK

Received 9 June 2007, revised 29 October 2007, accepted 6 December 2007

Published online 7 March 2008

PACS 02.70.Ns, 78.20.Bh, 78.20.Ci, 78.40.Dw

${ }^{*}$ Corresponding author: e-mail rg@soton.ac.uk, Phone: +44 02380 592649, Fax: +44 02380593781

We have used wavelength scanning close to the Brewster angle of water to determine the relative index of refraction between the surface film and the bulk liquid. The result is clearly that contrary to expectation, the surface film has a higher index than the bulk. This runs counter to molecular dynamics calculations which propose a tapering off of density, and by implication of refractive index. The finding may be more in line with recent suggestions that clusters of hydronium ions may dominate the structure close to the surface.
1 Introduction The surface film on water is of current interest to physicists and chemists alike, and has been since the early measurements of Lord Rayleigh [1] on reflection from transparent materials. A review of literature up to 2001 [2] is available. More recently, the chemistry of the interface has been the focus of molecular dynamics calculations [3]. This paper offers some physical evidence of one property of the interface, the refractive index, which may help to distinguish between alternative models. We use a method in which the Brewster angle of a transparent material is effectively scanned by applying the wavelength limits such that the switch in $\Delta$ from 180 to $0 / 360^{\circ}$ occurs midway through the scan. As a method, working close to the Brewster angle is usually ignored in the literature, because it can lead to too much sensitivity and noisy irreproducible results which may be difficult to interpret. In our case, the experiment and the substrate could not be simpler, and the method has advantages as will be shown.

2 Experimental The ellipsometer is the Uvisel from Horiba Jobin-Yvon, which has a Xe arc illuminator, and a scan range from 250 and $800 \mathrm{~nm}$. As well as the usual precautions about calibrating the monochromator and checking the alignment of the elllipsometer in the straightthrough position of the arms, it is critically important that the frame of the elllipsometer, and hence the goniometer and arm positioning mechanism is aligned with the true horizontal. As the surfaces of the liquid samples are necessarily horizontal, any deviation from this by the elllipsometer frame translates directly to azimuthal errors of the di- vided circles. Purified water was prepared by reverse osmosis using a Sartorius Arium-UV assembly.. Other chemicals were used as received. The experiments were carried out in a temperature-controlled room at $22 \pm 1{ }^{\circ} \mathrm{C}$.

3 Results and discussion Ellipsometrists usually avoid making measurements exactly at the Brewster angle. In mathematical terms, the Fresnel equations for a transparent material with no surface film, i.e. with an abrupt interface between the ambient and substrate, predict that at the Brewster angle the ellipsometric quantity $\Delta$ is undefined, and the quantity $\Psi$ is zero. Below the Brewster angle $\Delta$ is $180^{\circ}$ while above it $\Psi$ is finite and $\Delta$ is zero. In fact ellipsometry cannot distinguish between the angles $0^{\circ}$ and $360^{\circ}$.

As soon as there is a surface film, however thin it may be, this situation changes in that $\Delta$ in the neighbourhood of the Brewster angle will take finite values. There are then two distinguishable cases. Either the surface film (and we are assuming a very thin film) has an average refractive index greater than, or less than the substrate. Our experiments are designed to explore this angular dependence very close to the Brewster angle and so probe the index of the surface film relative to that of the bulk.

Instead of attempting to vary the angle of incidence (AOI) in extremely fine steps, we use the alternative technique of scanning the wavelength. Calculating the Brewster angle of the bulk at a particular wavelength in the middle of the available range, the goniometers are then set to and fixed at this value or slightly above it. The refractive 
index of all the materials used decreases slowly with increasing wavelength, which means that at short wavelengths the AOI is below the Brewster angle, and above it at long wavelengths,. Therefore the $\Delta$ value will be close to $180^{\circ}$ at short wavelengths, and $0^{\circ}$ or equivalently $360^{\circ}$ at long wavelengths. For an ideal material with an abrupt interface, a wavelength scan would therefore show a step function from $180^{\circ}$ either upwards to $360^{\circ}$ or downwards to $0^{\circ}$. More likely, given the unavoidable signal noise in $\Delta$, frequent transitions between the two extremities would be observed, as is the case in our results.

The main source of signal noise is vibration in the water surface, which is highly variable in intensity and very difficult to eliminate. At the extremities of the wavelength scan the signal strength is low, and detector noise dominates.

We looked at a series of water and other liquids including ethanol, dimethylsulfoxide (DMSO) and liquid paraffin (Figs. 1 and 2). In all other cases besides water, the step function is replaced by a more or less steep downward trajectory from $180^{\circ}$ to $0^{\circ}$. Water gives a slowly varying upward trend.

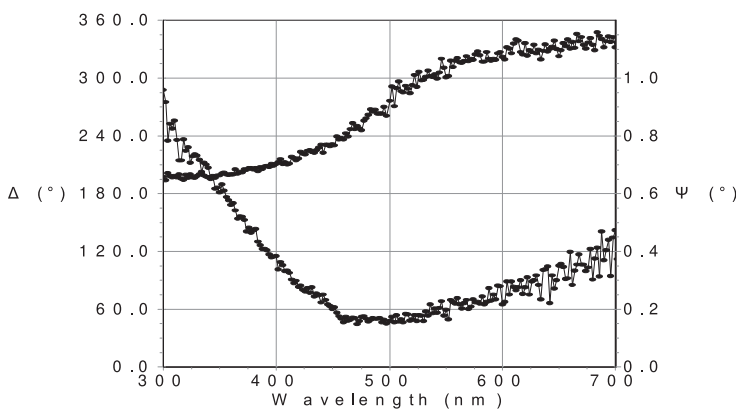

Figure 1 Water at AOI $52.9^{\circ}, \Delta$ (upper curve) and $\Psi$ (lower curve).

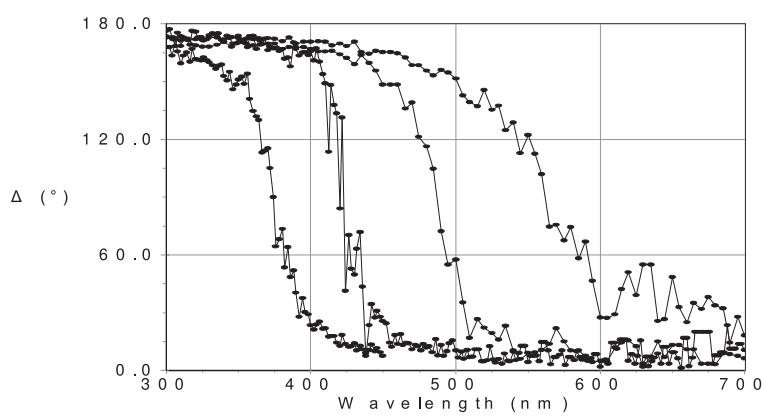

Figure $2 \Delta$ curves for various substances and AOI. From the left: Liquid paraffin $\left(56^{\circ}\right)$, DMSO $\left(55.8^{\circ}\right)$, Ethanol $\left(53.7^{\circ}\right)$, Dodecane $\left(54.7^{\circ}\right)$. Some noise-induced $0 / 360^{\circ}$ transitions have been omitted.

Clearly a model of the optical properties of the materials is required. Using the modelling software accompanying the Uvisel ellipsometer, various simple models were explored.
The surface forces controlling the liquid surface layer are necessarily short range, so the models were limited to a single surface film a maximum of a few tens of nm thick. The film was assumed to be uniform and have optical properties similar to those of the bulk.

The fundamental observation emerging is that if the average refractive index of the surface film is greater than that of the bulk the $\Delta$ trajectory is upwards towards $360^{\circ}$, and downwards for the reverse situation.

Being transparent, all the layers were represented by a three-term Sellmeier dispersion equation, thus keeping as low as possible the number of variables to be fitted. The quality of the fits is sensitive to data noise, and in general the error surfaces are shallow leading to high uncertainties in the parameters. Fig. 3 shows the agreement between fitted model and measured data. In Fig. 4 the wavelength dependence of the fitted model RI values is compared with those for the apparent RI for water assuming bulk values extending to the surface with no surface layer. Reference data for water obtained by a variety of non-surface dependent techniques are also shown [4].

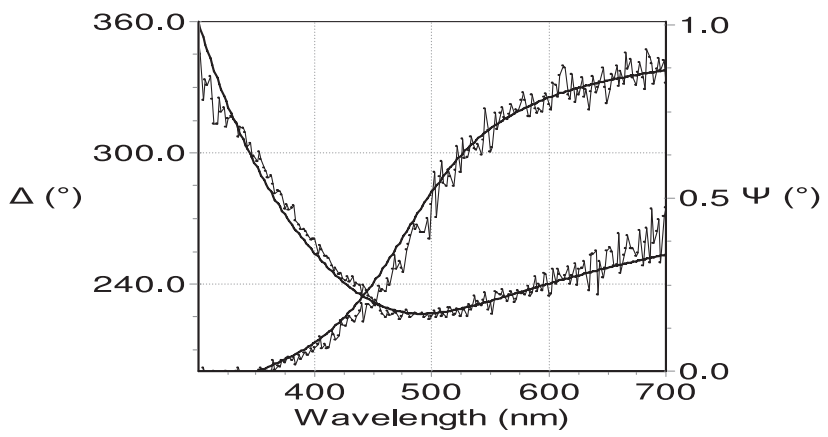

Figure 3 Measured data (connected points) and fit (smooth curves) for the single-film model for the surface film on water.

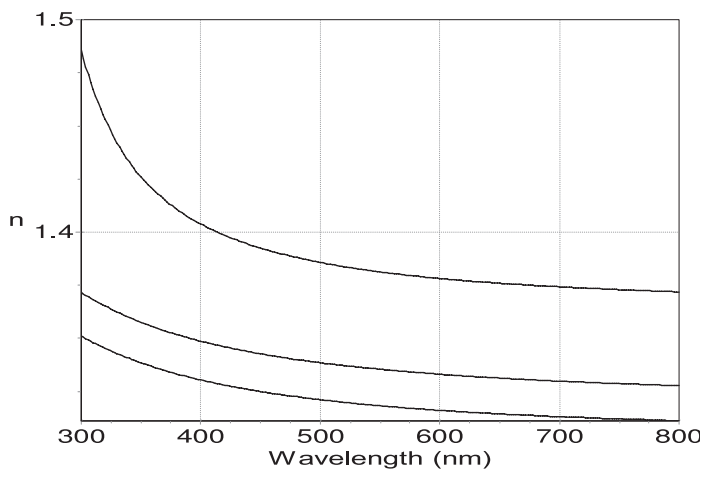

Figure 4 Fitted RI data for the bulk ellipsometric value of water assuming no surface layer (lowest curve), Reference data from [4] (middle curve) and fitted data from single-film model (top curve).

This fit generates a value of $(1.71 \pm 0.064) \mathrm{nm}$ for the thickness of the film. Further work is needed to establish the uniqueness of these fitted results. The Sellmeier equation is capable of representing transparent materials well, includ- 
ing bulk water and its surface film. However the parameters do not have physical meaning. The question remains as to whether a more physically meaningful dispersion equation can be found in the visible spectral range, which would allow more specific conclusions regarding the state of aggregation of the surface film. Certainly the refractive index data in the visible range can be represented by one or more classical oscillators, although it is equally difficult to assign physical processes to the parameters in this range. In contemplating whether the technique could be extended into the IR range to acquire more meaningful molecular structure parameters, it should be borne in mind that optically absorbing materials do not display a well-defined Brewster angle.

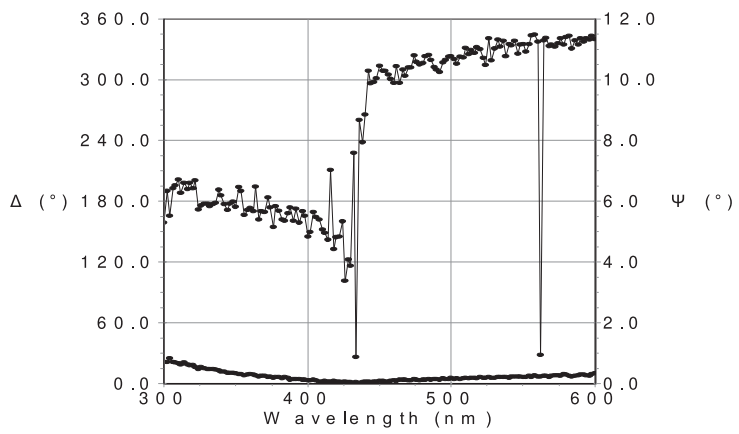

Figure $5 \Delta$ (upper curve) and $\Psi$ curves for water with 1 drop dilute detergent at AOI 52.9

At this stage a more promising approach may be to consider chemical interactions in the surface film (see related paper in this conference on dye adsorption at the water/air interface), particularly in view of the more specific chemical models that are now emerging from other investigations. Some preliminary experiments were done with a range of solutes including detergent and $\mathrm{KCl}$ (Figs. 5 and $6)$. The upward trend of $\Delta$ is resistant to even high concentrations of electrolytes, less so to ionic surfactants. Obviously more work is required to explore the effects of diverse solutes.

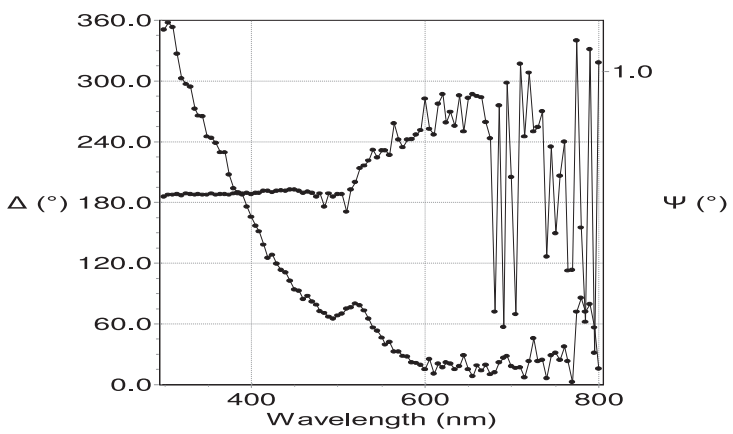

Figure $6 \Delta$ (upper curve) and $\Psi$ curves for $1 \mathrm{M} \mathrm{KCl}$ in water, AOI $53.1^{\circ}$.

Dyes were studied in more detail (see paper entitled "Adsorption of Rhodamine 6G at the water-air interface" in this conference). Just from this category of solutes alone, there is a new vista of diagnostic experiments opening up with which to account for the different surface behaviour of ionic and neutral dyes.

Even though the spectroscopic information this work has unfolded is not specific enough for a detailed picture of the molecular mechanisms at work in the interfacial layer, the findings are sufficiently dramatic that they should be included in any future work on the topic.

\section{Conclusion}

The experiments described here are very simple, but they have thrown new light, so to speak, on the nature of the water-air interface. In further work, attention should be paid to reducing mechanical vibrations which disturb the water surface, and to better optical signal enhancement. Longer integration times are useful for suppressing some kinds of detector noise, but runs cannot be extended too far without eliminating evaporation of the target material. Small height variations translate to angle of incidence changes, which cannot be tolerated in this ultra-sensitive regime.

\section{References}

[1] Lord Rayleigh, Philos. Mag. 33, 209 (1892).

[2] J. Penfold, Rep. Prog. Phys. 64, 777 (2001).

[3] V. Buch, A. Milet, R. Vacha, P. Jungwirth, and J.P. Devlin, Proc. Nat. Acad. Sci. 104, 7343 (2001).

[4] E. D. Palik, Handbook of Optical Constants of Solids (Academic Press, New York, 1980). 